\title{
Sorption to soil, biochar and compost: Is prediction to multicomponent mixtures possible based on single sorbent measurements?
}

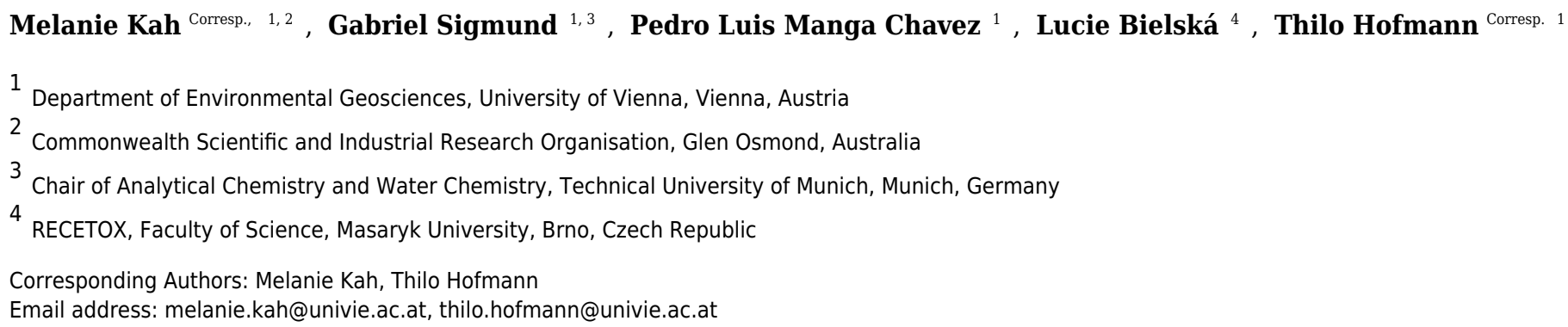

Amendment with biochar and/or compost has been proposed as a strategy to remediate soil contaminated with low levels of polycyclic aromatic hydrocarbons. The strong sorption potential of biochar can help sequestering contaminants while the compost may promote their degradation. An improved understanding of how sorption evolves upon soil amendment is an essential step towards the implementation of the approach. The present study reports on the sorption of pyrene to two soils, four biochars and one compost. Detailed isotherm analyses across a wide range of concentration confirmed that soil amendments can significantly increase the sorption of pyrene. Comparisons of data obtained by a classical batch and a passive sampling method suggest that dissolved organic matter did not play a significant role on the sorption of pyrene. The addition of 10 $\%$ compost to soil led to a moderate increase in sorption ( $<2$-fold), which could be well predicted based on measurements of sorption to the individual components. Hence, our result suggest that the sorption of pyrene to soil and compost can be relatively well approximated by an additive process. The addition of $5 \%$ biochar to soil (with or without compost) led to a major increase in the sorption of pyrene (2.5-4.7-fold), which was however much smaller than that suggested based on the sorption measured on the three individual components. Results suggest that the strong sorption to the biochar was attenuated by up to $80 \%$ in the presence of soil and compost, much likely due to surface and pore blockage. Results were very similar in the two soils considered, and collectively suggest that combined amendments with compost and biochar may be a useful approach to remediate soils with low levels of contamination. Further studies carried out in more realistic settings and over longer periods of time are the next step to evaluate the long term viability of remediation approaches based on biochar amendments. 


\section{Sorption to soil, biochar and compost: is prediction to}

\section{2 multicomponent mixtures possible based on single sorbent}

\section{3 measurements?}

5 Melanie Kaha ${ }^{\mathrm{a}, \mathrm{b}^{*}}$, Gabriel Sigmund ${ }^{\mathrm{a}, \mathrm{c}}$, Pedro Luis Manga Chavez ${ }^{\mathrm{a}}$, Lucie Bielskád ${ }^{\mathrm{d}}$, Thilo

6 Hofmann ${ }^{\mathrm{a}^{*}}$

7 a University of Vienna, Department of Environmental Geosciences and Environmental Science

8 Research Network, Althanstrasse 14, 5 UZA2, 1090 Vienna, Austria

$9 \quad{ }^{\mathrm{b}}$ Commonwealth Scientific and Industrial Research Organisation (CSIRO) Land \& Water, Waite

10 Campus, Locked Bag No 2, Glen Osmond, SA 5064, Australia

$11{ }^{c}$ Present address: Chair of Analytical Chemistry and Water Chemistry, Technical University of

12 Munich, Marchioninistrasse 17, 81377 Munich, Germany

$13{ }^{\mathrm{d}}$ RECETOX, Faculty of Science, Masaryk University, Kamenice 5, 62500 Brno, Czech

14 Republic

15

16 Melanie.kah@univie.ac.at, thilo.hofmann@univie.ac.at 


\section{Abstract}

Amendment with biochar and/or compost has been proposed as a strategy to remediate soil contaminated with low levels of polycyclic aromatic hydrocarbons. The strong sorption potential of biochar can help sequestering contaminants while the compost may promote their degradation. An improved understanding of how sorption evolves upon soil amendment is an essential step towards the implementation of the approach. The present study reports on the sorption of pyrene to two soils, four biochars and one compost. Detailed isotherm analyses across a wide range of concentration confirmed that soil amendments can significantly increase the sorption of pyrene. Comparisons of data obtained by a classical batch and a passive sampling method suggest that dissolved organic matter did not play a significant role on the sorption of pyrene. The addition of $10 \%$ compost to soil led to a moderate increase in sorption $(<2$-fold $)$, which could be well predicted based on measurements of sorption to the individual components. Hence, our result suggest that the sorption of pyrene to soil and compost can be relatively well approximated by an additive process. The addition of $5 \%$ biochar to soil (with or without compost) led to a major increase in the sorption of pyrene (2.5-4.7-fold), which was however much smaller than that suggested based on the sorption measured on the three individual components. Results suggest that the strong sorption to the biochar was attenuated by up to $80 \%$ in the presence of soil and compost, much likely due to surface and pore blockage. Results were very similar in the two soils considered, and collectively suggest that combined amendments with compost and biochar may be a useful approach to remediate soils with low levels of contamination. Further studies carried out in more realistic settings and over longer periods of time are the next step to evaluate the long term viability of remediation approaches based on biochar amendments. 


\section{Introduction}

40 Biochar is a carbon rich material produced by the pyrolysis of a variety of feedstock at temperatures below $700{ }^{\circ} \mathrm{C}(\mathrm{EBC}, 2012$; IBI, 2015). Biochar was originally produced as a soil

42

amendment with objectives including carbon sequestration, soil fertility improvement and byproduct/waste recycling (Ahmad et al., 2014). Many studies have shown that biochar can exhibit a high sorption affinity for a range of organic contaminants including polycyclic aromatic hydrocarbons (Cornelissen et al., 2005; Hale et al., 2011; Kah et al., 2016), polychlorinated biphenyls (Chai et al., 2012; Wang et al., 2013; Denyes, Rutter \& Zeeb, 2013) and ionisable compounds (Teixidó et al., 2011; Xiao \& Pignatello, 2015; Sigmund et al., 2016). Sorption is a key process to limit the bioavailability and transport of organic contaminants, and the incorporation of biochar into contaminated soil and sediment has thus been suggested as a remediation strategy to limit exposure and off-site transport of contaminants from contaminated sites (Ahmad et al., 2014)

Ideally, a remediation strategy does not only aim at sequestering and reducing the uptake of organic contaminants, but also considers their possible degradation into harmless products. The presence of biochar can reduce the degradation kinetics of organic contaminants in soils (Marchal et al., 2013), and the simultaneous addition of compost has been considered to counteract the undesired impact of biochar on degradation, while providing an additional source of organic matter. In particular, two recent studies have suggested that combining biochar and compost amendments to soils contaminated with polycyclic aromatic hydrocarbons may be successful to significantly reduce toxicity to soil organisms e.g. Caenorhabditis elegans (Bielská et al., 2017), while avoiding the full inhibition of the degradation process (Sigmund et al., 2018). Both studies suggested that changes in toxicity and degradation occurred through changes in the 
62 contaminant sorption, but details regarding the process were limited to data obtained at relatively

63 high concentrations and/or in the presence of multiple contaminants. More detailed

64 investigations into the sorption process are thus needed to support the future development of

65 remediation approaches combining biochar and compost amendments.

66 With the aim to gain more insights into the changes in sorption provoked by the addition of

67 combined amendments, the present study considers detailed investigations into the sorption characteristics of a series of soil, biochar and compost mixtures. Pyrene was selected as a model organic contaminant representative of polycyclic aromatic hydrocarbons. The study design directly builds on the results of Bielska et al. (2017) and Sigmund et al. (2018), and focuses on aspects identified as requiring further investigations.

Sorption was first measured on four different types of biochars produced from miscanthus and soft wood, either at 550 or $700^{\circ} \mathrm{C}$. The biochar with the highest sorption potential was further considered to systematically investigate sorption in two soils, one compost and one biochar, either alone or in two and three-phase mixtures. Soil, compost and biochar are expected to interact upon mixing, and the sorption properties of their mixture is likely to differ from the sum of their individual sorption properties. In particular, the strong sorption of biochar is expected to be reduced through surface fouling by soil and/or compost. One of the main objective of this study was to determine the extent to which sorption in mixtures deviates from a simple additive model. Another objective was to consider sorption across a wide range of concentration, and to distinguish the contributions of partitioning (into labile organic matter) and adsorption (at the

82 surface of carbonised surfaces of biochar) by carrying out an in-depth isotherm analysis.

83 Our study was also designed to consider the possible impact of dissolved organic matter on 84 sorption. Compost is typically very rich in organic matter ( $>20 \%$, Brinton, 2000), and may 
85 produce dissolved organic matter that can facilitate the transport and uptake of sorbed

86

87

88

89

90

91

92

93

94

95

96

97

98 contaminants. For instance, the results of Bielska et al. (2017) indicated a possible contribution of particle-bound pathways to toxicity. Sorption is typically measured using the batch method, which includes a phase separation step often based on centrifugation. Dissolved organic matter may not be fully separated from the aqueous phase by centrifugation. Conversely, passive sampling methods such as that based on polyoxymethylene (POM), only considers the freely dissolved fraction of pyrene and eliminate the possible contribution of dissolved organic matter on sorption. In the present study, the sorption of pyrene was measured with the batch method as well as by passive sampling. Comparing sorption coefficients derived by the two methods indicates whether dissolved organic matter plays a significant role on sorption.

Overall, our study aimed at gaining a better understanding of the sorption of pyrene in mixtures of soil, biochar and compost, across a wide range of contaminant concentrations, and accounting for the possible effect of dissolved organic matter. These aspects are key to the further development and implementation of remediation strategies based on combined amendments but they have not been systematically considered up to now.

\section{Material and methods}

\section{Sorbents}

Biochars produced from soft wood and miscanthus grass straw at pyrolysis temperatures of $550^{\circ} \mathrm{C}$ and $700{ }^{\circ} \mathrm{C}$ were purchased from the UK Biochar Research Centre (SWP550, SWP700, MSP550 and MSP700, respectively). A compost containing $11.4 \%$ organic carbon was kindly provided by fk Agrar Umweltservice (Pixendorf, Austria). Two top soils were sampled from agricultural fields in Austria: a sandy loam from Eschenau (Lower Austria) and a clay loam from Kaindorf (Styria). After sampling, the two soils were air dried, sieved at $<2 \mathrm{~mm}$ and stored in 
108 the dark. Soil samples were amended with compost and/or biochar (all air dried, see the details in

109 the sorption section), well mixed and conditioned for about 3 days at $20^{\circ} \mathrm{C}$. The mixtures were

110 then dried overnight at $80^{\circ} \mathrm{C}$, crushed with a pestle and mortar, and sieved at $<250 \mu \mathrm{m}$ to ensure

111 homogenisation before the sorption experiments. All materials were thoroughly characterised in

112 previous studies, including elemental analysis, organic carbon, $\mathrm{pH}$, specific surface area and pore

113 volume (Sigmund et al., 2017b; Bielská et al., 2017). All properties are presented in Table S1-S3

114 of the supporting information. Scanning electron microscope images were obtained for the four

115 biochars with an Inspect ${ }^{\mathrm{TM}} \mathrm{S} 50$ scanning electron microscope operating under high vacuum, 10

$116 \mathrm{kV}$ acceleration voltage equipped with a secondary electron Everhart- Thornley detector (Figure

117 S1 and S2) following sputtering of the samples with carbon.

\section{Chemicals and analysis}

119 Hexane and methanol were of residue analysis grade (Lab Scan, Ireland and Acros Organics,

120 Belgium). Pyrene (99.5\%) and pyrene-d10 (99.5\%) were purchased from Dr. Ehrenstorfer

$121 \mathrm{GmbH}$ (Germany). Pyrene was analysed by gas chromatography-mass spectrometry (GC-MS):

122 Agilent 7890A coupled to Agilent 5975C; HP-5MS fused silica column: $60 \mathrm{~m} \times 250 \mu \mathrm{m} \times 0.25$

$123 \mu \mathrm{m}, \mathrm{J} \& \mathrm{~W}$ Scientific; pulsed splitless mode; oven temperature of $55^{\circ} \mathrm{C}$ for $1 \mathrm{~min}$, then $10^{\circ} \mathrm{C} / \mathrm{min}$

124 up to $300^{\circ} \mathrm{C}$. Quantification was based on deuterated internal standards added to the samples

125 before extraction. The limit of detection was $0.0054 \mu \mathrm{g} / \mathrm{L}$ for the batch sorption experiment and

$1260.0002 \mu \mathrm{g} / \mathrm{L}$ for the passive sampling method (the concentration refers to that remaining in the

127 aqueous phase of the sorbent suspension after sorption).

\section{Sorption measurements}

129 Sorption of pyrene was first measured on the four biochars (SWP550, SWP700, MSP550 and

130 MSP700) and the compost. The biochar exhibiting the highest sorption potential was further used 
131 in the sorbent mixtures: compost + biochar $(2: 1)$ and for each soil: soil $+10 \%$ compost, soil +

$1325 \%$ biochar, and soil $+10 \%$ compost $+5 \%$ biochar (all in dry weight based). The rates were

133 selected to be consistent with previous studies considering combined amendments (Bielská et al.,

134 2017; Sigmund et al., 2018).Sorption was measured using two techniques: a classical batch and a

135 passive sampling method using POM. All sorption experiments were conducted at $20^{\circ} \mathrm{C}$ and

136 using aqueous background solutions containing $0.01 \mathrm{M} \mathrm{CaCl}_{2}$ and $0.385 \mathrm{mM} \mathrm{NaN}_{3}$ to inhibit

137 biological activity. Pyrene stock solutions were prepared in methanol, and the amount spiked

138 was kept below $0.1 \%$ in volume to minimize cosolvent effects on sorption. Controls and blanks

139 were included in all experiments and the sorbed concentrations were calculated by mass balance.

140 For the batch method, sorbent suspensions were prepared in $50 \mathrm{~mL}$ glass centrifuge tubes $(10 \mathrm{mg}$

141 of biochar, $20 \mathrm{mg}$ of compost, $200 \mathrm{mg}$ of soil or soil mixture in $40 \mathrm{~mL}$ of background solution)

142 and equilibrated for 48 hours by horizontal shaking at $125 \mathrm{rpm}$. Pyrene was then spiked (the

143 initial concentration ranged 1-50 $\mathrm{g} / \mathrm{L}$ ), before samples were return to shaking for 48 hours,

144 which was previously demonstrated to be sufficient to reach sorption pseudo-equilibrium (data

145 not shown). Samples were then centrifuged at $1000 \mathrm{~g}$ for $40 \mathrm{~min}, 30 \mathrm{~mL}$ of supernatant were

146 collected, and extracted three times with hexane after addition of the internal standard (Pyr- $\left.\mathrm{d}_{10}\right)$.

147 Extracts were combined, concentrated down to $1 \mathrm{~mL}$ under $\mathrm{N}_{2}$ flow and analyzed by GC-MS

148 (see the details above). Sorption was measured by batch at six different concentration levels for

149 each sorbent and in triplicates.

150 The POM method allows sampling the truly dissolved portion of pyrene and studying the

151 possible effects that dissolved organic matter may have on sorption measurements. The POM

152 method also allows measuring sorption down to lower concentrations than with the classical

153 batch. The method was previously described and validated for pyrene (Kah et al., 2011, 2016) 
154 and it is only briefly described here. Clean POM strips of about $100 \mathrm{mg}$ were preconditioned in

155 the background solution for 72 hours, before being added to sorbent suspensions prepared as

156 described for the batch method. The samples were then spiked with pyrene (the initial

157 concentration ranged $1-75 \mu \mathrm{g} / \mathrm{L}$ ), and shaken horizontally in the dark at $125 \mathrm{rpm}$ for 31 days in

158 order to achieve equilibrium (Kah et al., 2011). The POM strips were then removed from the

159 vials and wiped with a wet tissue. The internal standard (pyrene-d10) was added and the POM

160 strips were extracted with $20 \mathrm{~mL}$ of hexane by shaking for 3 days at $125 \mathrm{rpm}$ (Cornelissen \&

161 Gustafsson, 2004). Extracts were concentrated under $\mathrm{N}_{2}$ flow and analyzed by GC-MS. Sorption

162 was measured by the POM method at 5-6 concentration levels and in duplicates.

\section{Data analysis}

164 Sorption isotherms were fitted with the Freundlich model:

$165 \mathrm{C}_{\mathrm{s}}=\mathrm{K}_{\mathrm{f}} \times \mathrm{C}_{\mathrm{w}}{ }^{\mathrm{n}}$

166

167

168

169

170

171

172

173

174 The dual-mode model was also fitted to the sorption isotherms in order to quantify the

175 contributions of adsorption Qad $(\mu \mathrm{g} / \mathrm{kg})$ and partitioning Qp $(\mu \mathrm{g} / \mathrm{kg})$ : 
$176 \mathrm{C}_{\mathrm{s}}=\mathrm{Q}_{\mathrm{ad}}+\mathrm{Q}_{\mathrm{p}}=\left(\mathrm{Q}_{\max } * \mathrm{C}_{\mathrm{w}}\right) /\left(\mathrm{b}+\mathrm{C}_{\mathrm{w}}\right)+\mathrm{K}_{\mathrm{p}} * \mathrm{C}_{\mathrm{w}}$

177 where $\mathrm{Q}_{\max }(\mu \mathrm{g} / \mathrm{kg})$ is the adsorption maximum capacity, $\mathrm{b}$ is the affinity coefficient $(\mu \mathrm{g} / \mathrm{L})$ and $178 \mathrm{~K}_{\mathrm{p}}$ is the partitioning coefficient $(\mathrm{L} / \mathrm{kg})$.

179 All statistical analyses, curve fits and graphs were produced using GraphPad Prism 6 (GraphPad 180 Software Inc. 2016), with a significance level set to $\alpha=0.05$. 


\section{Results}

\section{Sorption of pyrene to four biochars}

183 Sorption isotherms of pyrene to the four biochars could be well fitted by the Freundlich model $184\left(0.92<\mathrm{r}^{2}<0.97\right.$, Figure 1, all parameters are presented in Table S4). The linearity parameter $\mathrm{n}$ ranged between 0.45 and 0.67 , which is characteristic of the highly nonlinear sorption of pyrene to carbonaceous sorbents (Kah et al., 2016). Nonlinearity increased with the pyrolysis temperature and was greater for the biochars derived from soft wood than from miscanthus $(\mathrm{p}<0.001)$.

The biochars produced from miscanthus sorbed significantly more pyrene than biochars produced from soft wood, which was unexpected in view of the greater $\mathrm{C} \%$, surface area and pore volume of the latter (Table S2). Sorption of organic sorbates generally increases with the biochar production temperature (Kah et al., 2016), and this was observed for soft wood, but not for miscanthus in our study. The discrepancy cannot be interpreted in terms of surface chemistry (e.g. aromaticity and polarity indices shown in Table S2) and neither in terms of physical parameters (surface area or porosity). Behazin et al. (2015) have previously shown that wood chip biochars exhibit atypical characteristics relative to miscanthus biochars, possibly related to

197 differences in their structure, as shown on the electron microscope images (Figure S1 and S2). Identifying the exact cause(s) for discrepancies in behaviour between the two feedstock materials will require further investigations. Biochar MS550, which exhibited the highest sorption for

200 pyrene, was considered for further investigations and it is designated as "biochar" in the 201 following discussion. 


\section{Comparison of the batch and POM methods}

203 We had hypothesised that the compost would generate dissolved organic matter that can sorb

204 significant amounts of pyrene. A previous study carried out with the same materials showed that

205 concentrations in dissolved organic carbon increased by $40 \%$ for the clay loam and $100 \%$ for the

206 sandy loam upon compost addition, reaching concentrations of about $850 \mathrm{mg} / \mathrm{kg}$ (details are

207 available in Bielska et al., 2017). Dissolved organic matter may not be fully separated from the

208 aqueous phase during the centrifugation step applied in the batch method, which can lead to $\mathrm{K}_{\mathrm{d}}$

209 batch $<\mathrm{K}_{\mathrm{d}}$ POM (the POM method only considers the freely dissolved portion of pyrene).

210 Figure S3 shows that the data generated by the batch and POM methods were consistent, and the

211 isotherms generated in the low (with POM) and higher concentration range (by batch)

212 constituted a continuous isotherm. When considering all sorbents, there was no significant

213 difference in the $K_{d}$ values derived by the two methods (paired t-tests on $K_{d}$ values calculated

214 based on isotherms fits at $0.05,0.08,0.2$ and $0.5 \mu \mathrm{g} / \mathrm{L}$, representing the range of concentration

215 where both methods could be applied). When differences were noticed, $\mathrm{K}_{\mathrm{d}}$ values generated by

216 batch tended to be greater than $\mathrm{K}_{\mathrm{d}}$ generated by POM, which goes against the hypothesis that

217 DOM played a significant role on the sorption of pyrene. It was previously demonstrated that

218 batch and POM methods can generate complementary results (Kah et al., 2011). In view of the

219 general continuity of the isotherm generated by batch and POM (Figure S3), the data were

220 combined. The following discussion is thus based on isotherm analysis across several orders of

221 magnitude.

\section{Sorption isotherms}

223 All sorption isotherms were very well fitted with the Freundlich model $\left(r^{2} \geq 0.95\right.$, Figure 2 and 3 ,

224 all parameters are presented in the Table S5, and isotherms are presented in logarithmic scale in 
225 Figure S4 and S5). The sorption affinity of pyrene to the clay loam was greater than to the sandy

226 loam, which is consistent with the higher organic carbon content of the former.

227 The sorption isotherm to the pure biochar was the most nonlinear $(n=0.67)$, while sorption to the 228 compost was one of the most linear $(n=0.89)$. The addition of compost to the biochar increased

229 the $n$ value from 0.67 to 0.73 and brought the isotherm close to that of the compost (Figure 2),

230 indicating that significant fouling of the biochar sorption sites by the compost must have

231 occurred (further discussed below). Similar observations were made with the soil mixture

232 presented in Figure 2: the linearity of the isotherms decreased upon the addition of biochar, and

233 increased upon addition of compost (either with or without biochar). Changes in the isotherm

234 linearity are consistent with previous literature, and reflect the contribution of partitioning to

235 labile organic matter from soil and compost (close to linear), relative to the contribution of

236 adsorption (nonlinear) to the carbonised surface of biochar (e.g. Schwarzenbach, Gschwend \&

237 Imboden, 2017; Sigmund et al., 2017b).

238 All sorption isotherms were also fitted with the dual mode model with the goal of distinguishing 239 and quantifying the respective contributions of absorption (mainly driven by the soil and

240 compost) and adsorption (mainly driven by the biochar). In many cases, the fits were ambiguous,

241 meaning that several set of values for the three parameters could equally well fit the curve. This

242 is likely because the dual mode model is too complex (over-parameterised) for the isotherms,

243 leading to highly unreliable values that should not be interpreted. The following discussion is

244 thus solely based on isotherm fits with the Freundlich model. Differences in sorption affinity

245 cannot be discussed based on $\mathrm{K}_{\mathrm{f}}$ values due to great differences in isotherm nonlinearity. Using

246 the isotherm parameters presented in Table S5, sorption coefficients were calculated at two

247 different concentrations and are discussed in the next section. 


\section{Impact of amendment mixtures on sorption: $K_{d}$ values}

249 Sorption coefficients calculated at $\mathrm{C}_{\mathrm{w}}=0.02$ and $2 \mathrm{mg} / \mathrm{L}\left(\mathrm{K}_{\mathrm{d}} 0.02\right.$ and $\left.\mathrm{K}_{\mathrm{d}} 2\right)$ are presented in Figure

2504 (grey crosses, all values are available in Table S5) to allow comparisons of sorption among

251 sorbents and their mixtures. Predictions of $\mathrm{K}_{\mathrm{d}}$ values for the mixtures were also computed based

252 on the $K_{d}$ value of pyrene to each individual sorbent, and their respective proportion in a given

253 mixture. The theoretical contributions of the soil, compost and biochar are represented by the

254 brown, green and black bars in Figure 4, respectively. The top of the bar indicates the $\mathrm{K}_{\mathrm{d}}$ value

255 predicted for the mixture by the additive model. For single sorbents, the prediction matches the

256 measurement (100\% contribution of one sorbent). For the mixtures, differences between the

257 predicted (bars) and measured $\mathrm{K}_{\mathrm{d}}$ values (crosses) indicate the extent to which sorption was

258 reduced through the interactions between sorbents.

259 Compost + Biochar

260 Consistently with isotherm nonlinearity, $K_{d} 0.02>>K_{d} 2$ for the biochar $(n=0.67)$, whereas

261 sorption varied only marginally with concentration for the compost $(n=0.89)$. In the low

262 concentration range, sorption to the mixture compost + biochar (2:1) was 2.7-fold greater than

263 sorption to compost alone. Despite the great increase measured upon biochar addition, $\mathrm{K}_{\mathrm{d}}$ value

264 in the mixture compost + biochar was 2.4-fold lower than that predicted based on the additive

265 model. Assuming that sorption to biochar was hindered by compost (but not vice versa), the data

266 suggests that sorption to biochar was reduced by $60 \%$ through interactions with the compost,

267 much likely involving surface coverage and pore blocking (Kwon \& Pignatello, 2005; Pignatello,

268 Kwon \& Lu, 2006; Oen et al., 2012). Trends were the same at the high concentration level,

269 though slightly attenuated (e.g. reduction in sorption by biochar by 55\%). 
270 Soil amendments with compost and biochar

271 The impact of pyrene concentration on sorption was the greatest in the soil + biochar mixtures

$272\left(\mathrm{~K}_{\mathrm{d}}\right.$ values were about 4-fold higher at $0.02 \mu \mathrm{g} / \mathrm{L}$ than at $\left.2 \mu \mathrm{g} / \mathrm{L}\right)$, which is consistent with the

273 strong isotherm nonlinearity. In the low concentration range, $\mathrm{K}_{\mathrm{d}}$ values increased in the order soil

$274<$ soil + compost $<$ soil + biochar + compost $<$ soil + biochar. The amendment of soil by biochar

275 increased the sorption of pyrene by a minimum of 2.5 fold, reflecting the powerful enhancing

276 effect that biochar can have on sorption, especially at low sorbate concentration. Soil

277 amendments with compost only had moderate effects, increasing the sorption by 1.2 and 1.6 fold

278 in the clay loam and in the sandy loam, respectively. As the concentration of pyrene increased,

$279 \mathrm{~K}_{\mathrm{d}}$ values followed the order soil $<$ soil + compost $\leq$ soil + biochar $<$ soil + compost + biochar,

280 which is consistent with the OC\% of the mixtures, and indicates that the specific sorption of

281 pyrene to biochar surfaces was progressively tampered by the presence of soil and compost.

282 Predictions of sorption based on the additive model (bars on Figure 4) overestimated the

283 measurements (crosses) in almost all cases, but the degree of discrepancy depended very much

284 on the type of sorbent mixture. Sorption of pyrene to the soil and to the compost is expected to

285 be mainly driven by partitioning phenomenon (Schwarzenbach, Gschwend \& Imboden, 2017)

286 and estimates of sorption based on the additive model gave reasonable estimates of the sorption

287 potential in the soil + compost mixtures (within a factor of 1.4 or less, see Figure 4). Conversely,

288 sorption affinity was generally greatly overestimated by the additive model in the mixtures

289 containing biochar, especially at low concentration of pyrene. For instance, sorption to the soil +

290 compost + biochar mixture was overestimated by about 3.5 times at $0.02 \mathrm{mg} / \mathrm{L}$. The sorption of

291 pyrene to pure biochar mainly consists in adsorption phenomena (typically $>90 \%$ ) and only very

292 little contributions of partitioning (Kah et al., 2016). The discrepancies between the predictions 
293 and measurements suggest that mixing the adsorption (biochar) and partitioning-dominated

294 phases (soil and compost) results in a transition from surface adsorption to partitioning processes

295 as soil and/or compost are added (as indicated by changes in isotherm nonlinearity). Following

296 the same reasoning as above, and assuming that sorption to biochar was hampered by the soil

297 and compost (but not vice versa), the data obtained at low concentration indicates that sorption to

298 biochar was attenuated by $81 \%$ and $84 \%$ in the presence of compost and clay loam or sandy

299 loam, respectively.

\section{Discussion}

301

302

303

304

305

306

307

308

309

310

311

312

313

314 for sediments, e.g. Hale et al., 2009). 
315 In our study, the sorption coefficients derived from an additive model based on data obtained at

316 high pyrene concentrations were within a factor 2 to the measurements in the soil + compost +

317 biochar mixtures. For very pragmatic purposes (e.g. range finding), our results thus indicate that

318 sorption of pyrene to mixtures may be predicted based on values measured for single sorbent at

319 high sorbate concentration (here $2 \mathrm{mg} / \mathrm{L}$ ). In the highest portion of the sorption isotherm, the

320 high energy sorption sites of biochars are unlikely to play a significant role, which in some way

321 accounts for the fouling of sorption sites by labile organic matter from e.g. soil and/or compost.

322 This approach should be applied with great care as it does not specifically account for the

323 processes discussed above, and it gives no indications about the bioavailability or desorption

324 potential of the contaminant.

325 Another practical observation emerging from our study is that the soil amendments had very

326 similar effects in the clay loam and the sandy loam, despite their different texture (15-27\% clay)

327 and OC content (1-1.7\%). Previous studies have shown that soil physico-chemical properties and

328 management practices (e.g. application rate and repetition) can affect the impact that biochar has

329 on the sorption of contaminants to soil (e.g. Kumari et al., 2014). Our results however indicate

330 that additions of similar quantities of compost and/or biochar could be advocated to achieve

331 contaminant sequestration in soils having properties within the range investigated here.

\section{Conclusions}

333 Detailed isotherm analyses across a wide range of concentration confirmed that soil amendment

334 with compost and biochar can significantly increase the sorption potential towards pyrene. The

335 dissolved organic matter of the compost did not seem to play a significant role on the sorption of

336 pyrene. Results were very similar in the two soils considered, and collectively suggest that 
337 combined amendments with compost and biochar may be a useful approach to remediate soils

338 that are contaminated with relatively low levels of polycyclic aromatic hydrocarbons.

339 The addition of compost to soil led to a moderate increase in sorption $(<2$-fold), which could be

340 well predicted based on measurements of sorption to the individual components. The results thus

341 indicate that sorption of pyrene to soil and compost can be relatively well approximated as being

342 additive phases dominated by partitioning phenomena. The addition of both compost and biochar

343 to soil led to a major increase in sorption (2.5-4.7-fold), which was however much smaller than

344 that suggested based on sorption to the three individual components. Results suggest that the

345 strong sorption to the biochar alone was attenuated by up to $84 \%$ in the presence of soil and

346 compost.

347 In realistic settings where biochar is amended to contaminated soil, biochar is subject to a range

348 of biological, physical and chemical processes involving its interactions with various soil

349 components, and leaching of its most soluble fractions (Hale et al., 2011; Wang et al., 2017).

350 These processes can have a direct impact on the surface chemistry and porosity of biochar and

351 the way it interacts with contaminants. The exact mechanisms involved and the role they play in

352 a given system remain mostly unknown. For instance, it has been suggested that ageing in soil

353 has a limited impact on the pore size distribution of some biochars (Sorrenti et al., 2016;

354 Sigmund et al., 2017a), but it is not known whether this applies to all biochar-soil systems. Our

355 results show that soil and compost can significantly reduce the sorption of contaminants to

356 biochar, and it has also been shown that pore blockage can entrap sorbed contaminants and

357 reduce their desorption potential (Yang \& Xing, 2007; Wang et al., 2017). How ageing and

358 weathering processes affect the remobilisation of contaminants (desorption) has been relatively

359 poorly considered up to now, although it an essential aspect impacting the long term 
360 performances of soil amendments. Lessons can be learnt from previous research carried out on

361 the treatment of contaminated sediments with activated carbon (e.g. Patmont et al., 2015), but

362 aspects specific to ageing processes occurring in soil certainly require further attention. More

363 realistic studies carried out over longer periods of time are needed to evaluate the long term

364 viability of soil remediation approaches based on biochar addition, with or without the addition

365 of compost.

\section{Acknowledgements}

367 We would like to thank Thorsten Hüffer for his help in designing the POM experiments, and

368 Vesna Micic for taking the electron microscope images (both University of Vienna). We are also

369 grateful to Gerhard Soja (Austrian Institute of Technology) for providing the soil samples. 


\section{References}

372 Ahmad M., Rajapaksha AU., Lim JE., Zhang M., Bolan N., Mohan D., Vithanage M., Lee SS., Ok YS. 2014. 373 Biochar as a sorbent for contaminant management in soil and water: A review. Chemosphere 99:19-23. DOI: 10.1016/j.chemosphere.2013.10.071.

375

376

377

378

Behazin E., Ogunsona E., Rodriguez-Uribe A., Mohanty AK., Misra M., Anyia AO. 2015. Mechanical, Chemical, and Physical Properties of Wood and Perennial Grass Biochars for Possible Composite Application. BioResources 11:1334-1348. DOI: 10.15376/biores.11.1.1334-1348.

Bielská L., Kah M., Sigmund G., Hofmann T., Höss S. 2017. Bioavailability and toxicity of pyrene in soils upon biochar and compost addition. Science of The Total Environment 595:132-140. DOI: 10.1016/j.scitotenv.2017.03.230.

Brinton WF. 2000.Compost quality standards and guidelines. Available at https://woodsend.com/wpcontent/uploads/2016/06/Brinton2000-International-Compost-Standards.pdf (accessed March $26,2018)$.

Chai Y., Currie RJ., Davis JW., Wilken M., Martin GD., Fishman VN., Ghosh U. 2012. Effectiveness of Activated Carbon and Biochar in Reducing the Availability of Polychlorinated Dibenzo-pdioxins/Dibenzofurans in Soils. Environmental Science \& Technology 46:1035-1043. DOI: 10.1021/es2029697.

Chen J., Chen W., Zhu D. 2008. Adsorption of nonionic aromatic compounds to single-walled carbon nanotubes: Effects of aqueous solution chemistry. Environmental Science and Technology 42:7225-7230. DOI: 10.1021/es801412j.

Cornelissen G., Gustafsson Ö. 2004. Sorption of Phenanthrene to Environmental Black Carbon in Sediment with and without Organic Matter and Native Sorbates. Environmental Science and Technology 38:148-155. DOI: 10.1021/es034776m. 
394 Cornelissen G., Gustafsson Ö., Bucheli TD., Jonker MTO., Koelmans AA., van Noort PCM. 2005. Extensive

395 Sorption of Organic Compounds to Black Carbon, Coal, and Kerogen in Sediments and Soils:

396 Mechanisms and Consequences for Distribution, Bioaccumulation, and Biodegradation. Environmental Science \& Technology 39:6881-6895. DOI: 10.1021/es050191b.

398

399

400

401

402

403

404

405

406

407

408

409

410

411

412

413

414

415

416

Denyes MJ., Rutter A., Zeeb BA. 2013. In situ application of activated carbon and biochar to PCB-

contaminated soil and the effects of mixing regime. Environmental pollution (Barking, Essex : 1987) 182:201-208. DOI: 10.1016/j.envpol.2013.07.016.

EBC. 2012.European Biochar Certificate - Guidelines for a Sustainable Production of Biochar. European Biochar Foundation (EBC), Arbaz, Switzerland. Version 6.3E of 14th August 2017, DOI:

10.13140/RG.2.1.4658.7043. Available at http://www.europeanbiochar.org/biochar/media/doc/ebc-guidelines.pdf (accessed March 14, 2018).

Hale S., Hanley K., Lehmann J., Zimmerman A., Cornelissen G. 2011. Effects of chemical, biological, and physical aging as well as soil addition on the sorption of pyrene to activated carbon and biochar. Environmental Science and Technology 45:10445-10453. DOI: 10.1021/es202970x.

Hale SE., Tomaszewski JE., Luthy RG., Werner D. 2009. Sorption of dichlorodiphenyltrichloroethane (DDT) and its metabolites by activated carbon in clean water and sediment slurries. Water Research 43:4336-4346. DOI: 10.1016/j.watres.2009.06.031.

Hou L., Zhu D., Wang X., Wang L., Zhang C., Chen W. 2013. Adsorption of phenanthrene, 2-naphthol, and 1-naphthylamine to colloidal oxidized multiwalled carbon nanotubes: Effects of humic acid and surfactant modification. Environmental Toxicology and Chemistry 32:493-500. DOI:

10.1002/etc. 2088 .

IBI. 2015.Standardized Product Definition and Product Testing Guidelines for Biochar That Is Used in Soil. Available at http://www.biochar- 
417

418

419

420

421

422

423

424

425

426

427

428

429

430

431

432

433

434

435

436

437

438

439

440

international.org/sites/default/files/IBI_Biochar_Standards_V2.1_Final.pdf (accessed March 14, 2018).

Kah M., Sun H., Sigmund G., Hüffer T., Hofmann T. 2016. Pyrolysis of waste materials: Characterization and prediction of sorption potential across a wide range of mineral contents and pyrolysis temperatures. Bioresource Technology 214:225-233. DOI: 10.1016/j.biortech.2016.04.091.

Kah M., Zhang X., Hofmann T. 2014. Sorption behavior of carbon nanotubes: Changes induced by functionalization, sonication and natural organic matter. Science of the Total Environment 497498:133-138. DOI: 10.1016/j.scitotenv.2014.07.112.

Kah M., Zhang X., Jonker MTO., Hofmann T. 2011. Measuring and modeling adsorption of PAHs to carbon nanotubes over a six order of magnitude wide concentration range. Environmental Science and Technology 45:6011-6017. DOI: 10.1021/es2007726.

Kumari KGID., Moldrup P., Paradelo M., Jonge LW de. 2014. Phenanthrene Sorption on BiocharAmended Soils: Application Rate, Aging, and Physicochemical Properties of Soil. Water, Air, \& Soil Pollution 225:2105. DOI: 10.1007/s11270-014-2105-8.

Kwon S., Pignatello JJ. 2005. Effect of Natural Organic Substances on the Surface and Adsorptive Properties of Environmental Black Carbon (Char): Pseudo Pore Blockage by Model Lipid Components and Its Implications for N2-Probed Surface Properties of Natural Sorbents. Environmental Science \& Technology 39:7932-7939. DOI: 10.1021/es050976h.

Li Q., Snoeyink VL., Mariñas BJ., Campos C. 2003. Pore blockage effect of NOM on atrazine adsorption kinetics of PAC: The roles of PAC pore size distribution and NOM molecular weight. Water Research 37:4863-4872. DOI: 10.1016/j.watres.2003.08.018.

Marchal G., Smith KEC., Rein A., Winding A., Wollensen DJ., Trapp S., Karlson UG. 2013. Impact of activated carbon, biochar and compost on the desorption and mineralization of phenanthrene in soil. Environmental Pollution 181:200-210. DOI: 10.1016/j.envpol.2013.06.026. 
441 Oen AMP., Beckingham B., Ghosh U., Kruså ME., Luthy RG., Hartnik T., Henriksen T., Cornelissen G. 2012.

442 Sorption of Organic Compounds to Fresh and Field-Aged Activated Carbons in Soils and Sediments. Environmental Science \& Technology 46:810-817. DOI: 10.1021/es202814e.

Patmont CR., Ghosh U., LaRosa P., Menzie CA., Luthy RG., Greenberg MS., Cornelissen G., Eek E., Collins J., Hull J., Hjartland T., Glaza E., Bleiler J., Quadrini J. 2015. In situ sediment treatment using activated carbon: A demonstrated sediment cleanup technology. Integrated Environmental Assessment and Management 11:195-207. DOI: 10.1002/ieam.1589.

Pignatello JJ., Kwon S., Lu Y. 2006. Effect of Natural Organic Substances on the Surface and Adsorptive Properties of Environmental Black Carbon (Char): Attenuation of Surface Activity by Humic and Fulvic Acids. Environmental Science \& Technology 40:7757-7763. DOI: 10.1021/es061307m.

Qiu Y., Xiao X., Cheng H., Zhou Z., Sheng GD. 2009. Influence of environmental factors on pesticide adsorption by black carbon: $\mathrm{pH}$ and model dissolved organic matter. Environmental Science and Technology 43:4973-4978. DOI: 10.1021/es900573d.

Schwarzenbach RP., Gschwend PM., Imboden DM. 2017. Environmental Organic Chemistry. WileyInterscience.

Sigmund G., Bucheli TD., Hilber I., Micić V., Kah M., Hofmann T. 2017a. Effect of ageing on the properties and polycyclic aromatic hydrocarbon composition of biochar. Environmental Science: Processes

Sigmund G., Hüffer T., Hofmann T., Kah M. 2017b. Biochar total surface area and total pore volume determined by $\mathrm{N} 2$ and $\mathrm{CO} 2$ physisorption are strongly influenced by degassing temperature. Science of the Total Environment 580:770-775. DOI: 10.1016/j.scitotenv.2016.12.023.

Sigmund G., Poyntner C., Piñar G., Kah M., Hofmann T. 2018. Influence of compost and biochar on 463 microbial communities and the sorption/degradation of PAHs and NSO-substituted PAHs in 
464

465

466

467

468

469

470

471

472

473

474

475

476

477

478

479

480

481

482

483

484

485

486

487

contaminated soils. Journal of Hazardous Materials 345:107-113. DOI:

10.1016/j.jhazmat.2017.11.010.

Sigmund G., Sun H., Hofmann T., Kah M. 2016. Predicting the Sorption of Aromatic Acids to Noncarbonized and Carbonized Sorbents. Environmental Science and Technology 50:3641-3648. DOI: 10.1021/acs.est.5b06033.

Sorrenti G., Masiello CA., Dugan B., Toselli M. 2016. Biochar physico-chemical properties as affected by environmental exposure. The Science of the Total Environment 563-564:237-246. DOI: 10.1016/j.scitotenv.2016.03.245.

Teixidó M., Pignatello JJ., Beltrán JL., Granados M., Peccia J. 2011. Speciation of the lonizable Antibiotic Sulfamethazine on Black Carbon (Biochar). Environmental Science \& Technology 45:1002010027. DOI: $10.1021 /$ es202487h.

Wang Y., Wang L., Fang G., Herath HMSK., Wang Y., Cang L., Xie Z., Zhou D. 2013. Enhanced PCBs sorption on biochars as affected by environmental factors: Humic acid and metal cations. Environmental Pollution 172:86-93. DOI: 10.1016/j.envpol.2012.08.007.

Wang B., Zhang W., Li H., Fu H., Qu X., Zhu D. 2017. Micropore clogging by leachable pyrogenic organic carbon: A new perspective on sorption irreversibility and kinetics of hydrophobic organic contaminants to black carbon. Environmental Pollution 220:1349-1358. DOI: 10.1016/j.envpol.2016.10.100.

Wu S., He H., Inthapanya X., Yang C., Lu L., Zeng G., Han Z. 2017. Role of biochar on composting of organic wastes and remediation of contaminated soils-a review. Environmental Science and Pollution Research 24:16560-16577. DOI: 10.1007/s11356-017-9168-1.

Xiao F., Pignatello JJ. 2015. $\pi+-\pi$ Interactions between (Hetero)aromatic Amine Cations and the Graphitic Surfaces of Pyrogenic Carbonaceous Materials. Environmental Science \& Technology 49:906-914. DOI: 10.1021/es5043029. 
488 Yang K., Xing B. 2007. Desorption of polycyclic aromatic hydrocarbons from carbon nanomaterials in water. Environmental Pollution 145:529-537. DOI: 10.1016/j.envpol.2006.04.020.

490

Zhang X., Kah M., Jonker MTO., Hofmann T. 2012. Dispersion state and humic acids concentration-

491 dependent sorption of pyrene to carbon nanotubes. Environmental Science and Technology

492 46:7166-7173. DOI: 10.1021/es300645m.

493 


\section{Figure 1 (on next page)}

Sorption of pyrene to four types of biochars produced from soft wood (circles) and miscanthus grass straw (squares) at $550^{\circ} \mathrm{C}$ and $700^{\circ} \mathrm{C}$ (SWP550, SWP700, MSP550 and MSP700, respectively).

The lines represent the fits with the Freundlich model (all fitted parameters are presented in Table S4). Graph (B) is a zoom of graph (A). 

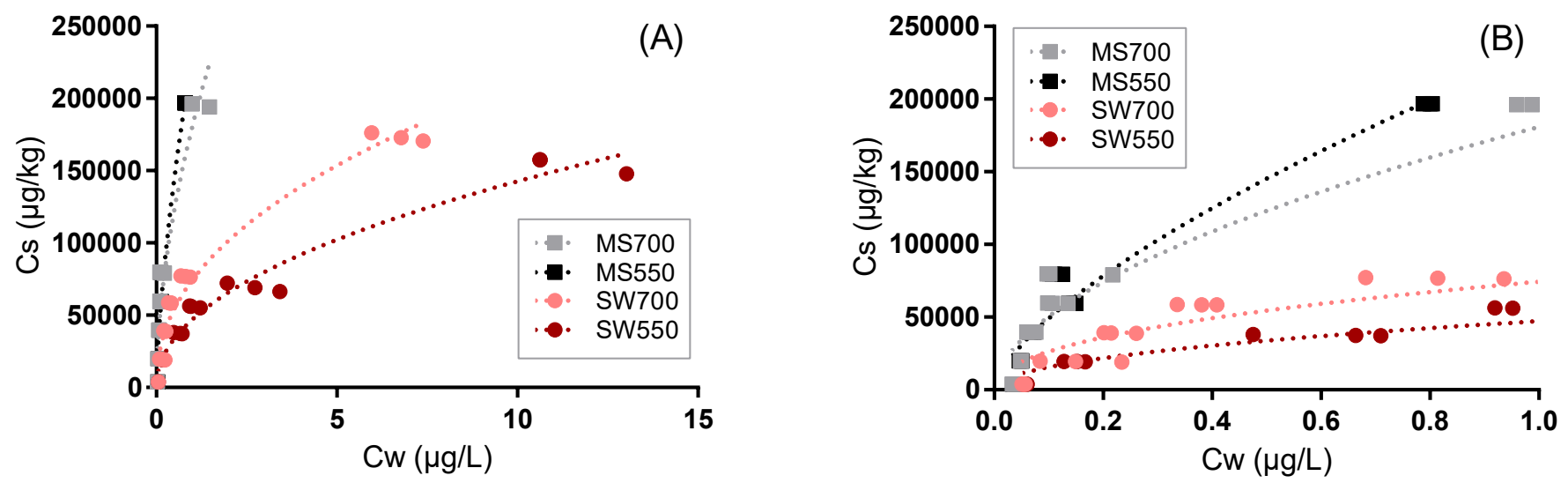
Figure 2 (on next page)

Sorption isotherms of pyrene to the biochar (black squares), compost (green triangles), and their mixture (1:2, green and black squares).

Graph (B) is a zoom of graph (A). 


\section{Biochar + Compost (A)}

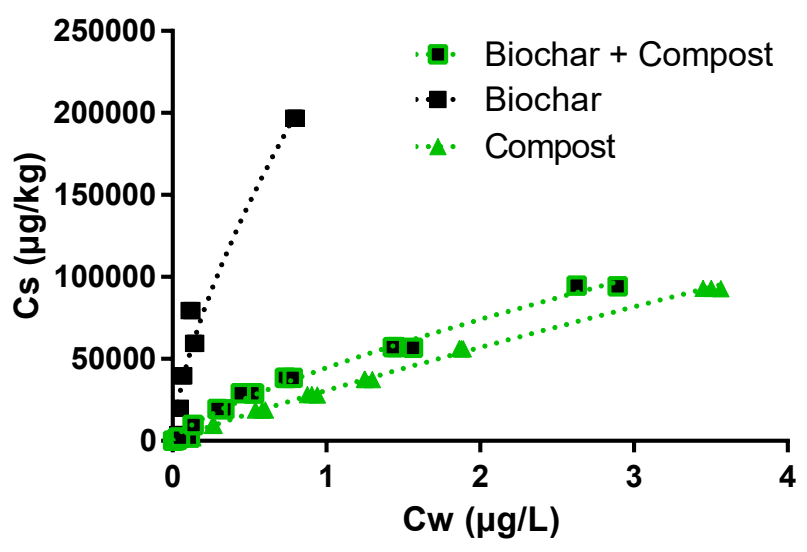

\section{Biochar + Compost (B)}

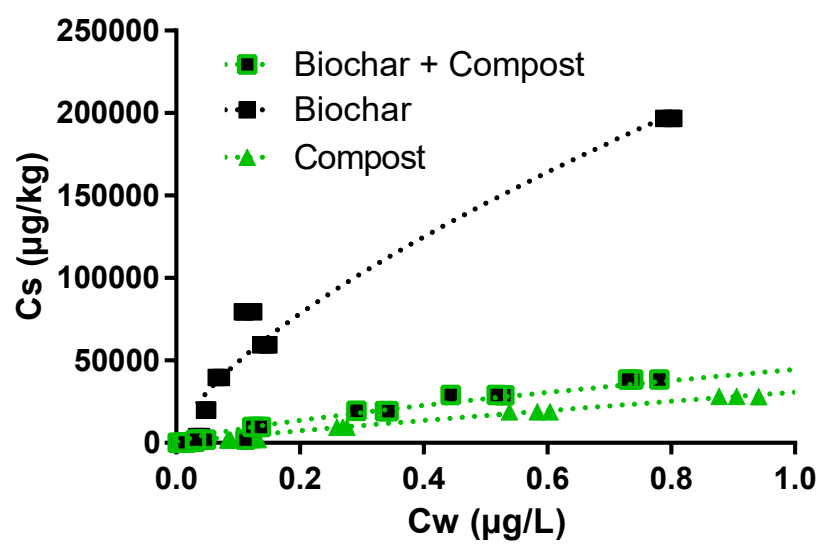


Figure 3 (on next page)

Sorption isotherms of pyrene to two soils before (open brown circles) and after addition of $5 \%$ biochar (black squares), $10 \%$ compost (green triangles) or both (full brown circles).

Graph (B) and (D) are zooms of graphs (A) and (C), respectively. Note that the highest concentrations measured for the soils alone are not shown, but they were considered in the isotherm fit. 


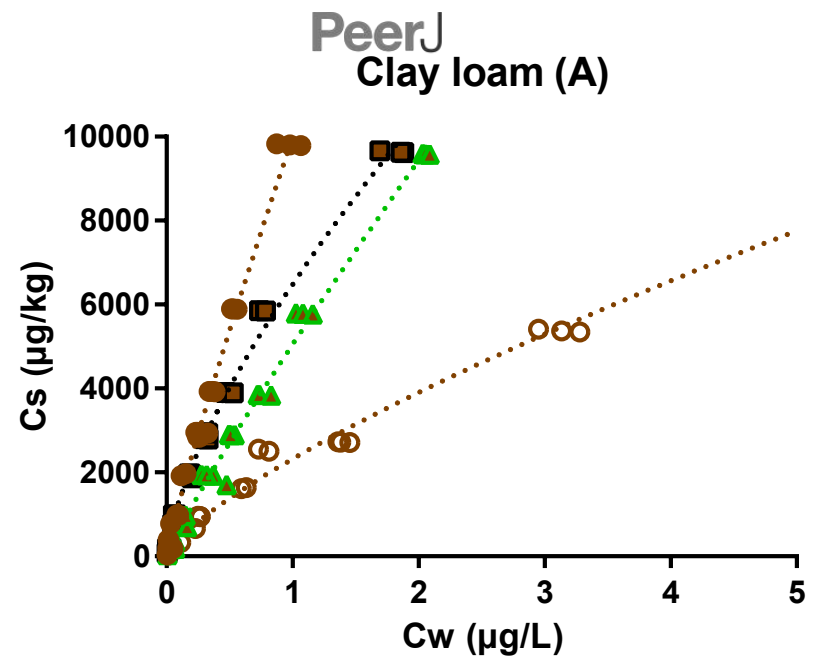

Clay loam (B)

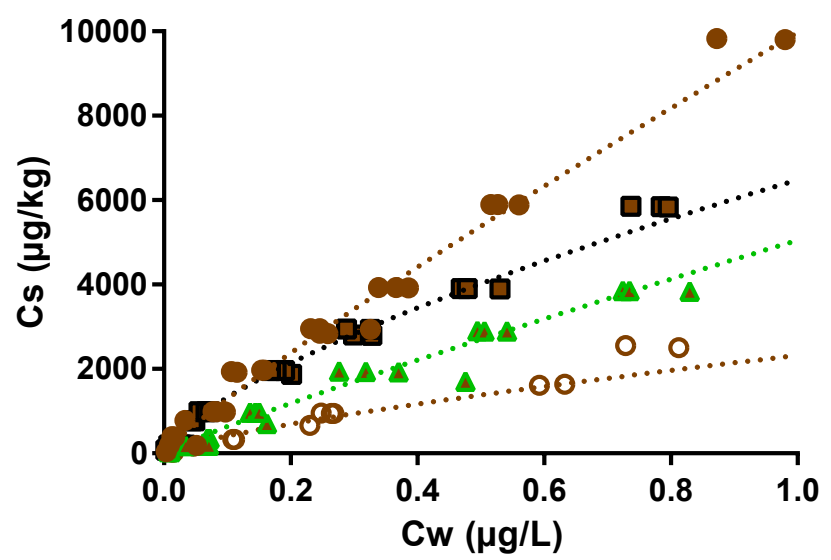

Sandy loam (C)

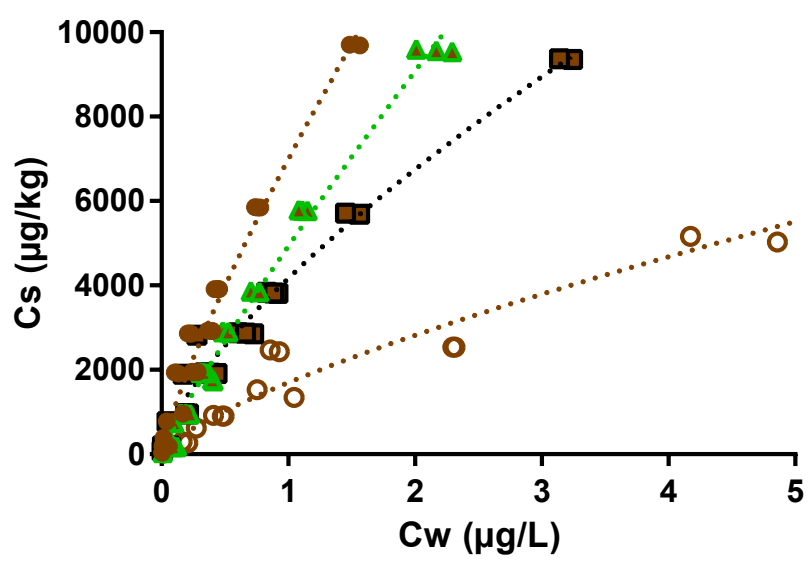

Sandy loam (D)

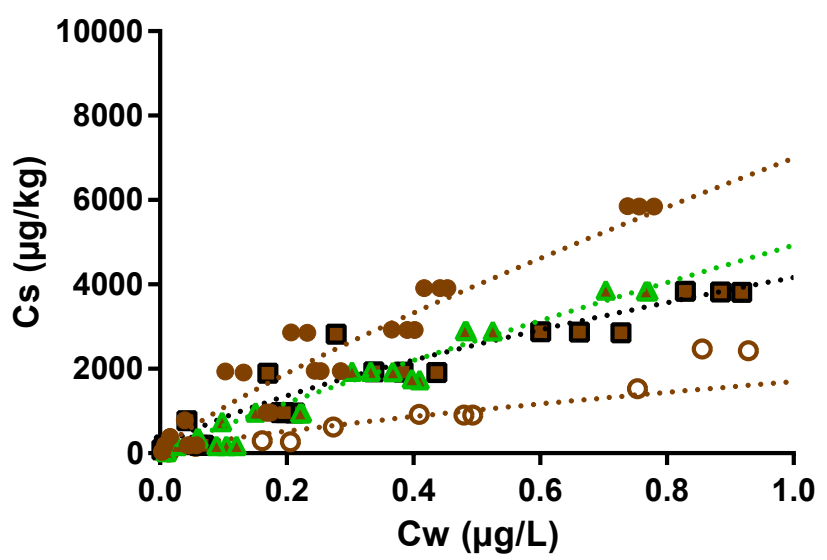

- - Soil + Biochar + Compost

- •. Soil + Biochar

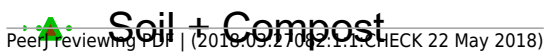

๑. Soil 
Figure 4 (on next page)

Measured sorption coefficients $(x)$ of pyrene at $0.02 \mathrm{mg} / \mathrm{L}(\mathrm{A})$ and $2 \mathrm{mg} / \mathrm{L}$ (B) compared to $K_{d}$ values calculated with an additive model (whole bars).

The bars show the contribution of soil (brown portion), compost ( $C$, green portion) and biochar (B, black portion). 


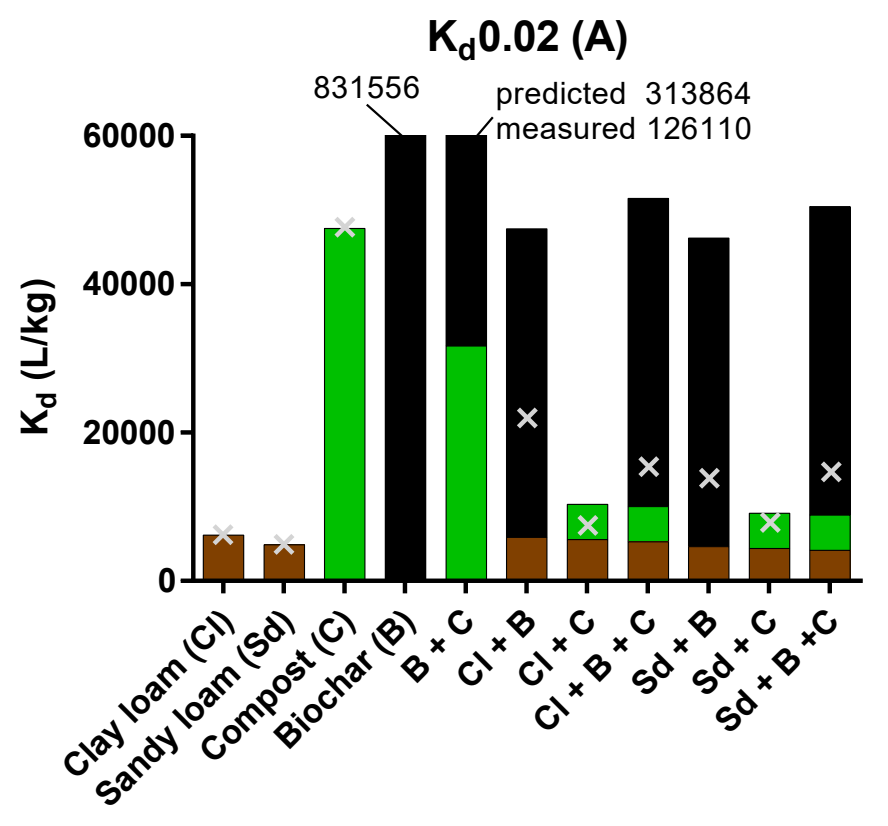

$K_{\mathrm{d}} 2$ (B)

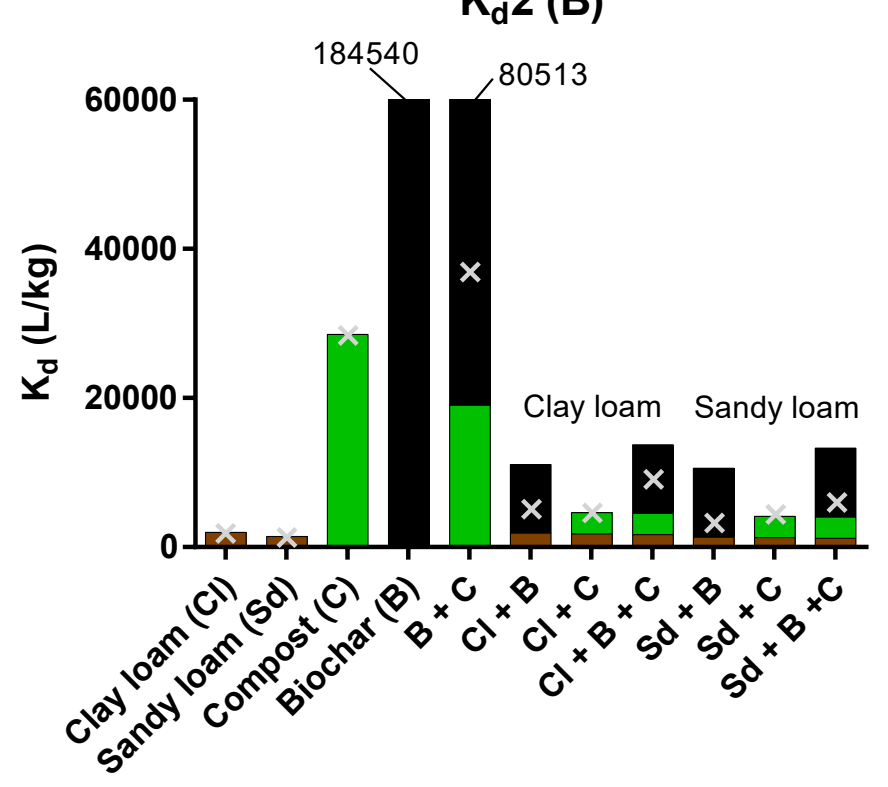

Measured in mixtures

Calculated based on individual components
- Biochar

$\square$ Compost

$\square$ Soil 